\title{
Identification of Myoferlin Expression for Prediction of Subsequent Primary Malignancy in Patients With Clear Cell Renal Cell Carcinoma
}

\author{
HYUN MIN KOH ${ }^{1}$, HYO JUNG AN ${ }^{1}$, GYUNG HYUCK KO ${ }^{2,3,4}$, JEONG HEE LEE ${ }^{2,3,4}$, \\ JONG SIL LEE ${ }^{2,3,4}$, DONG CHUL KIM ${ }^{2,3,4}$, DEOK HA SEO ${ }^{5}$ and DAE HYUN SONG ${ }^{1,2,3}$ \\ ${ }^{1}$ Department of Pathology, Gyeongsang National University Changwon Hospital, Changwon, Republic of Korea; \\ ${ }^{2}$ Gyeongsang National University School of Medicine, Jinju, Republic of Korea; \\ ${ }^{3}$ Gyeongsang Institute of Health Science, Jinju, Republic of Korea; \\ ${ }^{4}$ Department of Pathology, Gyeongsang National University Hospital, Jinju, Republic of Korea; \\ ${ }^{5}$ Department of Urology, Gyeongsang National University Changwon Hospital, Changwon, Republic of Korea
}

\begin{abstract}
Background/Aim: Multiple primary malignant tumors are common in patients with renal cell carcinoma. However, reports on the factors that can identify patients with a risk for subsequent primary malignancies have been lacking. This study aimed to investigate whether myoferlin expression can be used as a potential marker to predict subsequent primary malignancies in patients with clear cell renal cell carcinoma (ccRCC). Materials and Methods: We evaluated the relationship of subsequent primary malignancies with clinicopathological factors and myoferlin expression in 152 patients with $c c R C C$, and we analyzed the strength of the association with myoferlin expression. Results: The development of subsequent primary malignancies exhibited significant correlation with patient age $(p=0.029)$, sex $(p=0.015), T$ stage $(p<0.001)$, and myoferlin expression $(p=0.017)$. Furthermore, myoferlin hyperexpression was determined as an independent risk factor for developing a subsequent primary malignant tumor in patients with ccRCC (odds ratio(OR), 2.485, 95\% Confidence Interval $(C I)=1.052-5.870, p=0.038)$. Conclusion: Myoferlin hyperexpression can be a useful marker for predicting the development of subsequent primary malignancies in patients with ccRCC.
\end{abstract}

This article is freely accessible online.

Correspondence to: Dae Hyun Song, Department of Pathology, Gyeongsang National University School of Medicine, 79 Gangnamro, Jinju 52727, Republic of Korea. Tel: +82 552143150, Fax: +82 552143174, e-mail: golgy@hanmail.net

Key Words: Carcinoma, clear cell, kidney, myoferlin, subsequent primary malignancy.
Multiple primary malignant tumors are generally defined as two or more discrete cancers that are not the result of metastasis or recurrence, but are independent of each other $(1,2)$. According to the International Association of Cancer Registries and International Agency for Research on Cancer (IACR/IARC), one site is regarded as multiple primary malignancies (IARC 2004), not a single tumor of different parts of one site, and a 6-month period is recommended to distinguish between synchronous and metachronous multiple primaries (3).

Previous reports have shown that multiple primary malignant tumors are more common in patients with urogenital cancer, and a much higher prevalence of multiple tumors is associated with renal cell carcinoma $(\mathrm{RCC})(4,5)$. Moreover, Beisland et al. (2) have suggested that patients with RCC should be followed-up with examinations that are more general, due to their increased risk of developing other subsequent primary malignancies. However, little is known regarding the factors that can identify patients with a risk for subsequent primary malignancies.

Myoferlin is a member of the ferlin family, and it has been associated with cell membrane repair (6). Myoferlin is expressed in muscle cells and is involved in musculopathy (7). During the embryonic development of muscle or the regeneration of mature muscle cells following damage, myoblasts come together and form large syncytial myofibers (8). Myoferlin is highly expressed during myoblast fusion (8), however, the physiological functions of myoferlin remain to be determined (6).

Myoferlin hyper-expression has been studied in many cancers, such as gastrointestinal and hepatobiliary cancers, as well as in cancers of the female reproductive organs (9). Moreover, several reports have shown that myoferlin hyperexpression correlates with poor prognosis in pancreatic and 
oropharyngeal squamous cell carcinomas $(6,10,11)$. In the same context, we have previously found that myoferlin hyperexpression significantly correlates with high Fuhrman nuclear grade and poorer disease-free survival in patients with clear cell renal cell carcinoma (ccRCC) (6). Furthermore, Blomme et al. (12) have reported that myoferlin can be considered as a valuable cancer-derived exosomal protein, and myoferlin-lacking exosomes do not contribute as much to tumor cell migration and proliferation as exosomes that have myoferlin. As cancer-derived exosomes are considered to be released, not only in the vicinity of the cancer, but in the systemic areas as well, we hypothesized that systemically released myoferlin from cancer-derived exosomes affects host immunity and contributes to a pro-cancer microenvironment with epidemiological factors of cancers, resulting in an increased risk of developing cancer $(3,13)$.

Therefore, in this study, we evaluated whether myoferlin expression can be used as a potential marker for predicting subsequent primary malignancies in patients with ccRCC.

\section{Materials and Methods}

Patients and clinicopathological data. Samples from 152 consecutive patients who underwent treatment for sporadic ccRCC between January 2000 and December 2009 at Gyeongsang National University Hospital (Jinju, Korea) were examined by two experienced pathologists. All of the included patients had undergone nephrectomy. Staging was performed according to the American Joint Committee on Cancer TNM Classification of Malignant Tumors, eighth edition (7). Clinical data from the patients were collected retrospectively through examination of their medical records. Subsequent primary malignancies were defined as metachronous tumors detected in an interval of more than 6 months arising at different sites, according to the IACR/IARC, and were diagnosed by pathological or radiological confirmation.

This study was approved by the Institutional Review Board of Gyeongsang National University Hospital (2015-12-001) and was conducted in accordance with the principles embodied in the Declaration of Helsinki (6).

Tissue microarray construction. Hematoxylin and eosin -stained slides as well as formalin-fixed paraffin-embedded tissue blocks were reviewed, and the most representative tumor area was marked. Two cores ( $2 \mathrm{~mm}$ in diameter) of the tumor area were obtained from each specimen of representative Fuhrman nuclear grades (6).

Immunohistochemistry. Immunohistochemistry was performed on 4$\mu \mathrm{m}$-thick sections from tissue microarray (TMA) blocks. Tissues were stained with anti-myoferlin primary antibody at a dilution of 1:100 (7D6, Abcam, UK) as described previously (7), using an automated immunostainer (Benchmark Ultra, Ventana Medical Systems Inc., Tucson, AZ, USA). The endothelial cells of intratumoral vascular channels were used as positive control (14).

Myoferlin expression. Immunoreactivity of the tumor cells was evaluated in both the membrane and cytoplasm by visual counting
Table I. Clinicopathological information of patients.

\begin{tabular}{lc}
\hline Factor & Number $(\%)$ \\
\hline Mean age (years) & 59.9 \\
Male gender & $109(71.7)$ \\
T stage & \\
1a & $91(59.9)$ \\
$1 \mathrm{~b}$ & $24(15.8)$ \\
$2 \mathrm{a}$ & $9(5.9)$ \\
$2 \mathrm{~b}$ & $3(2.0)$ \\
$3 \mathrm{a}$ & $21(13.8)$ \\
$3 \mathrm{~b}$ & $2(1.3)$ \\
4 & $2(1.3)$ \\
Fuhrman nuclear grade & \\
1 & $26(17.1)$ \\
2 & $102(67.1)$ \\
3 & $19(12.5)$ \\
4 & $5(3.3)$ \\
Subsequent primary malignancy & \\
Absent & $139(91.4)$ \\
Present & $13(8.6)$ \\
Myoferlin expression (cores) & \\
Hypoexpression & $233(76.6)$ \\
Hyperexpression & $71(23.4)$ \\
Total & 152 patients \\
& 304 cores \\
\hline
\end{tabular}

(Figure 1). The stained tumor cells were graded as either hypoexpression or hyperexpression. Tumor cells that displayed stronger myoferlin expression compared to the membrane or the cytoplasm of the endothelial cells and stained more than $50 \%$ of tumor cells were graded as hyperexpression (6), otherwise, they were graded as hypoexpression. If tumor cells revealed heterogeneous expression of myoferlin in the same core, the representative value was decided according to the majority of tumor cells (6). To confirm reproducibility, all samples were graded by two independent pathologists in a blinded manner. If discrepancies occurred, a consensus was reached.

Statistical analysis. To determine the correlation of subsequent primary malignancy with clinicopathological factors and myoferlin expression, Pearson's chi-square test was used. To evaluate the strength of the association between myoferlin expression and the development of subsequent primary malignancies, a logistic regression analysis was performed (15). A $p$-value of equal or less than 0.05 was considered statistically significant. The analysis was performed using IBM SPSS version 24.0 (IBM Corp., Armonk, NY, USA).

\section{Results}

Clinicopathological information of patients with ccRCC. Clinicopathological information of the patients with ccRCC is shown in Table I. The mean age of the patients was 59.9 years (range: 32 to 83 years). T stages of the tumors of the patients were as follows: i) 1a in $91(59.9 \%)$, ii) $1 \mathrm{~b}$ in 24 $(15.8 \%)$, iii) $2 \mathrm{a}$ in $9(5.9 \%)$, iv) $2 \mathrm{~b}$ in $3(2.0 \%)$, v) $3 \mathrm{a}$ in 21 


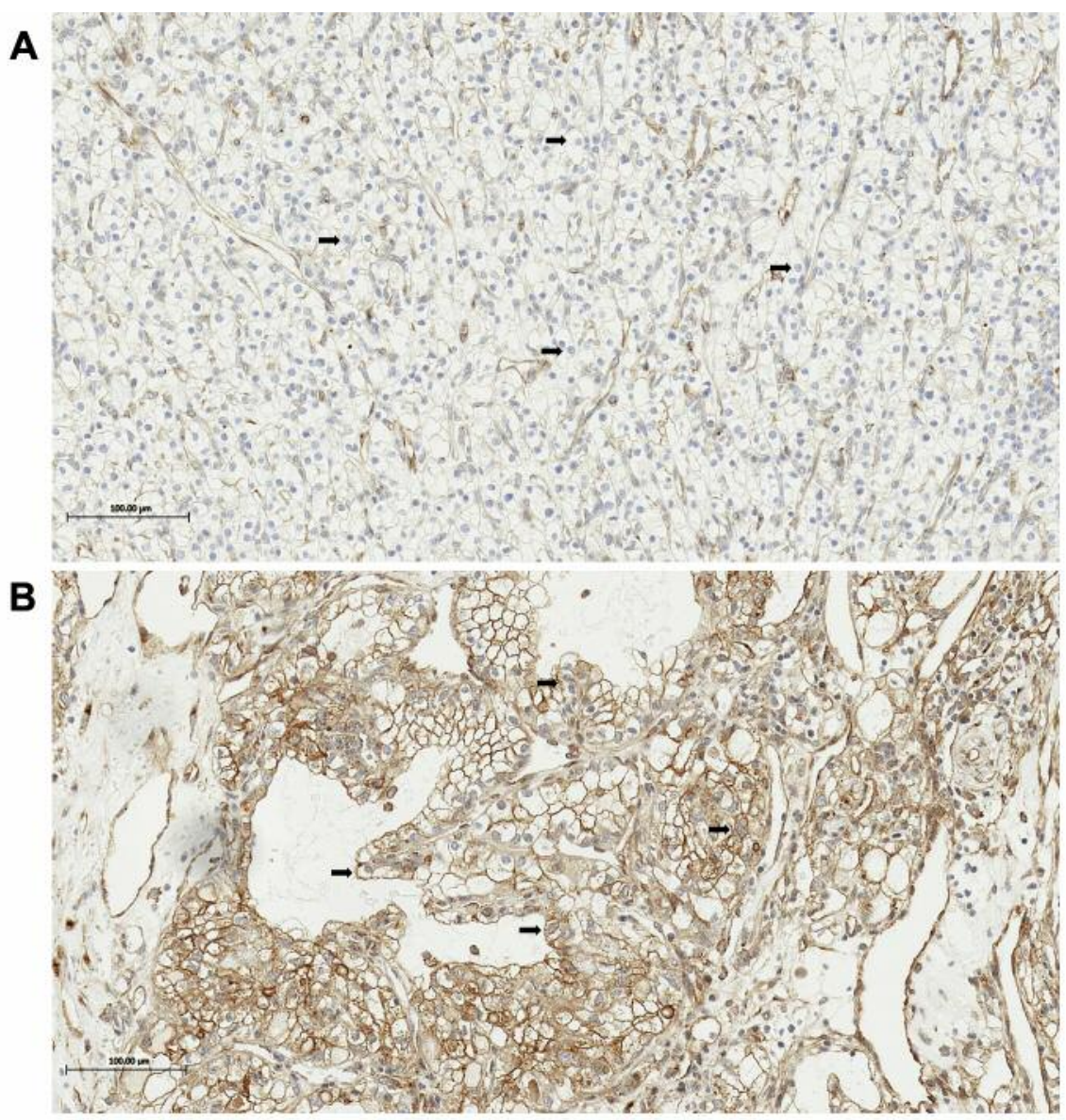

Figure 1. Myoferlin expression in clear cell renal cell carcinoma. The tumor cells show hypoexpression of Myoferlin (light brown color, arrows) (A), and hyperexpression of Myoferlin (brown color, arrows) (B). Scale bars: $100 \mu \mathrm{m}$.

$(13.8 \%)$, vi) $3 \mathrm{~b}$ in $2(1.3 \%)$, and vii) 4 in $2(1.3 \%)$. The Fuhrman nuclear grades were as follows: i) $26(17.1 \%)$ were grade 1 , ii) $102(67.1 \%)$ were grade 2 , iii) $19(12.5 \%)$ were grade 3 , and iv) $5(3.3 \%)$ were grade 4 . The myoferlin expression profiles were as follows: 233 cores (76.6\%) displayed hypoexpression, while the remaining 71 cores (23.4\%) displayed hyperexpression (6). Among all patients recruited, 13 patients $(8.6 \%)$ had subsequent primary malignancies.

Clinicopathological information of ccRCC patients with subsequent primary malignancy. Clinicopathological information of the patients with subsequent primary malignancy is shown in Table II. The mean age of the patients was 66.5 years (range: 50 to 81 years). Most of the patients were male $(12,92.3 \%)$. T stages of the tumors of the patients were as follows: i) $1 \mathrm{a}$ in $5(38.5 \%)$, ii) $1 \mathrm{~b}$ in 1 $(7.7 \%)$, iii) $2 \mathrm{a}$ in $1(7.7 \%)$, iv) $2 \mathrm{~b}$ in $1(7.7 \%)$, and v) $3 \mathrm{a}$ in $5(38.5 \%)$. The Fuhrman nuclear grades were as follows: i)
$1(7.7 \%)$ was grade 1 , ii) $9(69.2 \%)$ were grade 2 , and iii) 3 $(23.1 \%)$ were grade 3 . The myoferlin expression profiles were as follows: 15 cores (57.7\%) displayed hypoexpression, while the remaining 11 cores (42.3\%) displayed hyperexpression. Among the subsequent primary tumors, 3 (23.1\%) were hepatocellular carcinoma, 2 (15.4\%) were lung cancer, and the remaining 8 cases were adenocarcinoma of the pancreas, common bile duct, stomach, colon and prostate, neuroendocrine carcinoma of the pancreas, adenocarcinoma in situ of the stomach, and urothelial carcinoma of the ureter. The mean duration between ccRCC and subsequent primary malignancy was 3.6 years (range: 1 to 10.5 years).

Relationship of subsequent primary malignancy with clinicopathological factors and myoferlin expression. The relationship of subsequent primary malignancy with clinicopathological factors and myoferlin expression is shown in Table III. Subsequent primary malignancy significantly correlated with patient age $(p=0.029)$, sex 
in vivo $33: 1103-1108(2019)$

Table II. Clinicopathological information of patients with a subsequent primary malignancy ( $n=13)$.

\begin{tabular}{|c|c|c|c|c|c|c|}
\hline $\begin{array}{l}\text { No. of } \\
\text { patients }\end{array}$ & Gender/Age & T Stage & $\begin{array}{l}\text { Furhman nuclear } \\
\text { grade }\end{array}$ & $\begin{array}{l}\text { Myoferlin expression } \\
\text { (core 1/core 2) }\end{array}$ & $\begin{array}{c}\text { Duration* } \\
\text { (years) }\end{array}$ & $\begin{array}{c}\text { Subsequent primary } \\
\text { malignancy }\end{array}$ \\
\hline 1 & $\mathrm{M} / 64$ & $1 \mathrm{a}$ & 2 & Hyper/hyper & NA & Neuroendocrine carcinoma of pancreas \\
\hline 2 & $\mathrm{M} / 74$ & $1 \mathrm{a}$ & 2 & Hyper/hypo & 3.6 & Hepatocellular carcinoma \\
\hline 3 & $\mathrm{M} / 67$ & $1 \mathrm{a}$ & 2 & Hyper/hypo & 4.2 & Adenocarcinoma of prostate \\
\hline 4 & $\mathrm{~F} / 72$ & $1 \mathrm{a}$ & 2 & Нyро/hyро & 2.8 & Adenocarcinoma in situ of stomach \\
\hline 5 & $\mathrm{M} / 69$ & $1 \mathrm{a}$ & 2 & Hypo/hypo & 4.7 & Hepatocellular carcinoma \\
\hline 6 & $\mathrm{M} / 58$ & $1 \mathrm{~b}$ & 2 & Hyper/hypo & 5.4 & Hepatocellular carcinoma \\
\hline 7 & $\mathrm{M} / 50$ & $2 \mathrm{a}$ & 1 & Hyper/hyper & 1.1 & Adenocarcinoma of colon \\
\hline 8 & $\mathrm{M} / 73$ & $2 \mathrm{~b}$ & 2 & Hypo/hypo & 1.9 & Lung cancer \\
\hline 9 & $\mathrm{M} / 81$ & $3 a$ & 3 & Hyper/hyper & 1.8 & Adenocarcinoma of pancreas \\
\hline 10 & $\mathrm{M} / 52$ & $3 a$ & 3 & Hyper/hyper & 2.8 & Lung cancer \\
\hline 11 & $\mathrm{M} / 75$ & $3 a$ & 2 & Hypo/hypo & 1.0 & Adenocarcinoma of stomach \\
\hline 12 & $\mathrm{M} / 65$ & $3 a$ & 2 & Hypo/hypo & 10.5 & Urothelial carcinoma of ureter \\
\hline 13 & $\mathrm{M} / 65$ & $3 \mathrm{a}$ & 3 & Hypo/hypo & NA & Adenocarcinoma of common bile duct \\
\hline
\end{tabular}

*Duration means the diagnostic period between clear cell renal cell carcinoma and subsequent primary malignancy. NA, not applicable.

$(p=0.015)$, and T stage $(p<0.001)$, but not with Fuhrman nuclear grade $(p=0.313)$. Moreover, increased myoferlin expression showed a significant association with subsequent primary malignancy $(p=0.017)$.

Logistic regression analysis of subsequent primary malignancy. The univariate analysis demonstrated that age [odds ratio (OR), 2.763; 95\% Confidence Interval (CI), 1.0777.090], $\mathrm{T}$ stage $(\mathrm{OR}=4.239,95 \% \mathrm{CI}=1.863-9.646)$, and myoferlin expression $(\mathrm{OR}=2.664,95 \% \mathrm{CI}=1.163-6.103)$ had a significant contribution to the occurrence of subsequent primary malignancy in patients with ccRCC. A multivariate analysis confirmed that myoferlin hyperexpression was an independent and significant risk factor for developing a subsequent primary malignant tumor in patients with ccRCC $(\mathrm{OR}=2.485,95 \% \mathrm{CI}=1.052-5.870, p=0.038)$ (Table IV). Moreover, higher $\mathrm{T}$ stage ( stage $\geq 2$ ) was determined to be a risk factor for developing subsequent primary malignancies $(\mathrm{OR}=3.674,95 \% \mathrm{CI}=1.584-8.523, p=0.002)$ (Table IV).

\section{Discussion}

This study investigated whether myoferlin expression can be used to predict the occurrence of a subsequent primary malignant tumor in patients with ccRCC. The development of subsequent primary malignancy revealed a significant correlation with patient age $(p=0.029)$, sex $(p=0.015)$, T stage $(p<0.001)$, and myoferlin expression $(p=0.017)$, but not with Fuhrman nuclear grade $(p=0.313)$. Furthermore, myoferlin hyperexpression was determined to be an independent risk factor for developing a subsequent primary malignant tumor in patients with ccRCC. This is the first report to associate myoferlin hyperexpression with a higher risk for developing
Table III. Relationship of subsequent primary malignancy with clinicopathological factors and myoferlin expression ( $n=304$ cores).

\begin{tabular}{|c|c|c|c|}
\hline & \multicolumn{2}{|c|}{ Subsequent primary malignancy } & \multirow[b]{2}{*}{$p$-Value } \\
\hline & Absent & Present & \\
\hline Age & & & 0.029 \\
\hline$<59$ & $126(95.5)$ & $6(4.5)$ & \\
\hline$\geq 59$ & $152(88.4)$ & $20(11.6)$ & \\
\hline Gender & & & 0.015 \\
\hline Male & $194(89.0)$ & $24(11.0)$ & \\
\hline Female & $84(97.7)$ & $2(2.3)$ & \\
\hline $\mathrm{T}$ stage & & & $<0.001$ \\
\hline$<2$ & $218(94.8)$ & $12(5.2)$ & \\
\hline$\geq 2$ & $60(81.1)$ & $14(18.9)$ & \\
\hline Fuhrman nuclear grade & & & 0.313 \\
\hline$<3$ & $235(92.2)$ & $20(7.8)$ & \\
\hline$\geq 3$ & $43(87.8)$ & $6(12.2)$ & \\
\hline Myoferlin expression & & & 0.017 \\
\hline Hypoexpression & 218 (93.6) & $15(6.4)$ & \\
\hline Hyperexpression & $60(84.5)$ & $11(15.5)$ & \\
\hline
\end{tabular}

Values are presented as number $(\%)$.

subsequent primary malignancies in patients with ccRCC. Consequently, we assert that the ccRCC patients with myoferlin hyperexpression should be followed up with more meticulous examinations for subsequent primary malignancies to provide early detection and treatment.

Based on these results, we presume that systemically released myoferlin affects host immunity and contributes to a pro-cancer microenvironment with epidemiologic factors of cancers, resulting in the increased risk for developing cancer $(3,13)$. However, further research is still needed for a more definitive answer. 
Table IV. Logistic regression model of subsequent primary malignancy for patients with clear cell renal cell carcinoma.

\begin{tabular}{|c|c|c|c|c|c|c|}
\hline \multirow[b]{2}{*}{ Variables } & \multicolumn{3}{|c|}{ Univariate analysis } & \multicolumn{3}{|c|}{ Multivariate analysis } \\
\hline & OR & $95 \% \mathrm{CI}$ & $p$-Value & OR & $95 \% \mathrm{CI}$ & $p$-Value \\
\hline Age $(<59 v s . \geq 59)$ & 2.763 & $1.077-7.090$ & 0.035 & 2.372 & $0.901-6.240$ & 0.080 \\
\hline T stage $(<2 v s . \geq 2)$ & 4.239 & $1.863-9.646$ & 0.001 & 3.674 & $1.584-8.523$ & 0.002 \\
\hline Fuhrman nuclear grade $(<3 v s . \geq 3)$ & 1.640 & $0.622-4.319$ & 0.317 & & & \\
\hline Myoferlin expression (hypo vs. hyper) & 2.664 & $1.163-6.103$ & 0.020 & 2.485 & $1.052-5.870$ & 0.038 \\
\hline
\end{tabular}

CI: Confidence interval; OR: odds ratio.

Interestingly, higher $\mathrm{T}$ stage (stage $\geq 2$ ) was determined to be a risk factor for developing subsequent primary malignancies in patients with ccRCC. This may be related to various predisposing factors, including the treatment of a previous malignancy, and the increased checkup frequency of cancer patients (3). However, herein we emphasize that the ccRCC patients with higher $\mathrm{T}$ stage should also be followed up in the same manner as patients with myoferlin hyperexpression because $\mathrm{T}$ stage was not significantly associated with myoferlin expression according to our previous study (6)

Previous reports have shown that multiple primary malignant tumors related to RCC can develop in various organs, including prostate, bladder, lung, breast, and colon $(2,4,16,18)$. Several researchers have advocated that malignant melanoma and non-Hodgkin's lymphoma are also associated with RCC (19). The rate of occurrence of multiple primary malignancies with RCC, including antecedent, synchronous, and subsequent tumors has been found at 4.5$26.9 \%$, and a quarter to almost half of these tumors were detected subsequently $(2,16,17,19)$. In this study, the incidence of subsequent primary malignancies with ccRCC was at $8.6 \%$, and the mean diagnostic period between ccRCC and subsequent primary malignancy was 3.6 years. The two most common subsequent primary tumors were hepatocellular carcinoma and lung cancer, and the higher incidence rate was shown for the hepatobiliary system, followed by the gastrointestinal tract.

There are inevitable limitations to this study as a retrospective analysis from a single center. For example, we could not assess the survival probability related to developing subsequent primary malignancies due to the small sample size. Larger cohorts and an organized study design are recommended in future studies.

In conclusion, our study identified that myoferlin hyperexpression can be a useful marker for predicting subsequent primary malignancies, and higher $\mathrm{T}$ stage (stage $\geq 2$ ) may be a risk factor for developing subsequent primary malignancies in patients with ccRCC.

\section{Conflicts of Interest}

The Authors declared no potential conflicts of interest.

\section{Authors' Contributions}

Conception and design was performed by HMK, HJA and DHS. Development of methodology was done by HMK and HJA. Acquisition of data was done by HMK, GHK, JHL, JSL, DCK and DHS, while analysis and interpretation of data was done by HMK, HJA, GHK, JHL, JSL, DCK and DHS. Writing, review and/or revision of the manuscript were done by HMK, HJA and DHS. Administrative, technical, or material support was provided by HMK, JHL, JSL and DHS. Study supervision was done by HMK, GHK, DCK, DHS

\section{Acknowledgements}

The Authors received no financial support for the research.

\section{References}

1 Howe HL: A review of the definition for multiple primary cancers in the United States. Workshop Proceedings from December 4-6, 2002, in Princeton, New Jersey. Springfield (IL): North American Association of Central Cancer Registries, May 2003. Available from: http://citeseerx.ist.psu.edu/viewdoc/ download?doi=10.1.1.466.3479\&re $p=$ rep $1 \&$ type $=$ pdf

2 Beisland C, Talleraas O, Bakke A and Norstein J: Multiple primary malignancies in patients with renal cell carcinoma: a national population-based cohort study. BJU Int 97: 698-702, 2006. PMID: 16536756. DOI: $10.1111 /$ j.1464-410X.2006. 06004.x

3 Vogt A, Schmid S, Heinimann K, Frick H, Herrmann C, Cerny $\mathrm{T}$ and Omlin A: Multiple primary tumours: challenges and approaches, a review. ESMO open 2: e000172, 2017. PMID: 28761745. DOI: 10.1136/esmoopen-2017-000172

4 Nakata S, Kato Y, Sato J, Mayuzumi T, Kumasaka F and Shimizu T: Analysis of multiple primary cancers in patients with urologic cancer. Inter J Clin Oncol 2: 40-46, 1997. DOI: 10. 1007/BF024925965.

5 Matzkin H and Braf Z: Multiple primary malignant neoplasms in the genitourinary tract: occurrence and etiology. J Urol 142: 1-12, 1989. PMID: 2659818. 
6 Song DH, Ko GH, Lee JH, Lee JS, Yang JW, Kim MH, An HJ Kang MH, Jeon KN and Kim DC: Prognostic role of myoferlin expression in patients with clear cell renal cell carcinoma. Oncotarget 8: 89033-89039, 2017. PMID: 29179496. DOI: 10.18632/oncotarget.21645

7 Song DH, Ko GH, Lee JH, Lee JS, Lee GW, Kim HC, Yang JW, Heo RW, Roh GS, Han SY and Kim DC: Myoferlin expression in non-small cell lung cancer: Prognostic role and correlation with VEGFR-2 expression. Oncol Lett 11: 998-1006, 2016. PMID: 26893682. DOI: 10.3892/ol.2015.3988

8 Doherty KR, Cave A, Davis DB, Delmonte AJ, Posey A, Earley JU, Hadhazy M and McNally EM: Normal myoblast fusion requires myoferlin. Development 132: 5565-5575, 2005. PMID: 16280346. DOI: $10.1242 /$ dev.02155

9 Eisenberg MC, Kim Y, Li R, Ackerman WE, Kniss DA and Friedman A: Mechanistic modeling of the effects of myoferlin on tumor cell invasion. Proc Natl Acad Sci USA 108: 2007820083, 2011. PMID: 22135466. DOI: 10.1073/pnas.1116327108

10 Kumar B, Brown NV, Swanson BJ, Schmitt AC, Old M, Ozer E, Agrawal A, Schuller DE, Teknos TN and Kumar P: High expression of myoferlin is associated with poor outcome in oropharyngeal squamous cell carcinoma patients and is inversely associated with HPV-status. Oncotarget 7: 18665-18677, 2016. PMID: 26919244. DOI: 10.18632/oncotarget.7625

11 Wang WS, Liu XH, Liu LX, Lou WH, Jin DY, Yang PY and Wang XL: iTRAQ-based quantitative proteomics reveals myoferlin as a novel prognostic predictor in pancreatic adenocarcinoma. J proteomics 91: 453-465, 2013. PMID: 23851313. DOI: 10.1016/j.jprot.2013.06.032

12 Blomme A, Fahmy K, Peulen O, Costanza B, Fontaine M, Struman I, Baiwir D, de Pauw E, Thiry, M, Bellahcene A, Castronovo V and Turtoi A: Myoferlin is a novel exosomal protein and functional regulator of cancer-derived exosomes. Oncotarget 7: 83669-83683, 2016. PMID: 27845903. DOI: 10.18632/oncotarget.13276
13. Blackwell RH, Foreman KE and Gupta GN: The role of cancerderived exosomes in tumorigenicity \& epithelial-tomesenchymal transition. Cancers 9: E105, 2017. PMID: 28796 150. DOI: $10.3390 /$ cancers 9080105

14 Bernatchez PN, Sharma A, Kodaman P and Sessa WC: Myoferlin is critical for endocytosis in endothelial cells. Am J of Physiol Cell Physiol 297: C484-92, 2009. PMID: 19494235. DOI: 10.1152/ajpcell.00498.200815

15 Pestova K, Koch AJ, Quesenberry CP, Shan J, Zhang Y, Leimpeter AD, Blondin B, Sitailo S, Buckingham L, Du J, Fei H and Van Den Eeden SK: Identification of fluorescence in situ hybridization assay markers for prediction of disease progression in prostate cancer patients on active surveillance. BMC Cancer 18: 2, 2018. PMID: 29291731. DOI: 10.1186/s12885-017-3910-4

16 Kantor AF, McLaughlin JK, Curtis RE, Flannery JT and Fraumeni JF Jr: Risk of second malignancy after cancers of the renal parenchyma, renal pelvis, and ureter. Cancer 58: 11581161, 1986. PMID: 3731042.

17 Sato S, Shinohara N, Suzuki S, Harabayashi T and Koyanagi T: Multiple primary malignancies in Japanese patients with renal cell carcinoma. Int J Urol 11: 269-275, 2004. PMID: 15147541. DOI: $10.1111 / \mathrm{j} .1442-2042.2004 .00792 . \mathrm{x}$

18 Di Silverio F, Sciarra A, Flammia GP, Mariani M and De Vico A: Multiple primary tumors: 17 cases of renal-cell carcinoma associated with primary tumors involving different steroid-hormone target tissues. World J Urol 15: 203-209, 1997. PMID: 9228728.

19 Rabbani F, Grimaldi G and Russo P: Multiple primary malignancies in renal cell carcinoma. J Urol 160: 1255-1259, 1998. PMID: 9751330. 